\title{
Clinical Study \\ Spectrum of Maternofetal Outcomes during Dengue Infection in Pregnancy: An Insight
}

\author{
Swati Sharma, Sandhya Jain, and Shalini Rajaram \\ Department of Obstetrics and Gynaecology, University College of Medical Sciences and Guru Tegh Bahadur Hospital, \\ New Delhi 110095, India \\ Correspondence should be addressed to Swati Sharma; drsharmaswati@rediffmail.com
}

Received 31 October 2015; Revised 15 February 2016; Accepted 28 February 2016

Academic Editor: Bryan Larsen

Copyright (C) 2016 Swati Sharma et al. This is an open access article distributed under the Creative Commons Attribution License, which permits unrestricted use, distribution, and reproduction in any medium, provided the original work is properly cited.

Dengue is a vector transmitted viral infection; tropical and subtropical countries see outbreaks of dengue each year. There is a paucity of literature on effects of dengue infection on pregnancy outcome and this prompted us to undertake a study for better understanding of pregnancy implications with dengue infection. Pregnant women admitted during the seasonal outbreak of dengue between September 2015 and October 2015 were studied and maternal and fetal outcomes in sixteen NS1Ag positive women were analysed. Out of sixteen women diagnosed with dengue fever, three had dengue shock syndrome (DSS) and eight had dengue haemorrhagic fever (DHF). The most common obstetric complication seen in $43 \%$ of the cases was oligohydramnios. Bleeding manifestations occurred in seven women and there were three maternal deaths. Perinatal complications included three intrauterine deaths, six nursery admissions, and one neonatal death. Thus dengue infection was associated with high maternal and perinatal mortality. In view of poor obstetric outcomes, this viral infection warrants early admission and prompt management.

\section{Introduction}

Dengue is a mosquito-borne viral disease. It is caused by viruses of the genus Flavivirus, family Flaviviridae, and Group IV ssRNA. Dengue is transmitted to humans by the mosquito Aedes aegypti and is one of the most rapidly spreading viral infections. There has been a 30 -fold increase in its incidence in the last 50 years coupled to increasing migration from rural to urban areas. Annually 50 million people acquire dengue infection worldwide [1]. Dengue remains a major health concern for Southeast Asian countries with cyclic epidemics. In India multiple viral serotypes are circulating and some regions have case fatality rates of 3$5 \%$ in general population, which is much higher than other Southeast Asian regions (1\%) [2].

Complications of dengue in pregnancy have been scarcely studied. Literature suggests an increased risk of maternal hemorrhage, preterm labour, and oligohydramnios [3-5]. Clinical presentation of dengue may be confused with HELLP syndrome, both conditions having elevated liver enzymes, haemolysis, and low platelet counts; however serology helps in distinguishing the two conditions. Low platelet counts pose a risk of postpartum hemorrhage. As reported in previous studies, dengue is associated with preterm births, low birth weight, fetal deaths, and vertical transmission leading to neonatal thrombocytopenia requiring platelet transfusions [6-10].

In a quest to answer vital issues we critically analysed dengue antigen positive cases in our institute and studied maternal and fetal outcomes.

\section{Material and Method}

This study was conducted in the Department of Obstetrics and Gynaecology of a tertiary level government hospital, Delhi, India. During the months of September and October 2015, 60 pregnant women were seen in the emergency room with complaint of fever and were admitted for evaluation. As per protocol, dengue PCR (NS1Ag) was done in all women. Out of 60, 16 were dengue positive.

Dengue was diagnosed using NS1Ag and/or IgM serology. Clinical grading was done according to WHO classification and case definitions. An acute febrile illness with two or more clinical manifestations like headache, retroorbital 
TABLE 1: Clinical profile of NS1Ag positive patients.

\begin{tabular}{|c|c|c|c|c|c|c|c|c|}
\hline & Parity & $\begin{array}{c}\text { POG } \\
\text { weeks + } \\
\text { days }\end{array}$ & $\operatorname{IgM}$ & Bleeding manifestations & $\begin{array}{l}\text { Obstetrics } \\
\text { complication }\end{array}$ & $\begin{array}{c}\text { ICU } \\
\text { admission }\end{array}$ & $\begin{array}{l}\text { Maternal } \\
\text { morbidity }\end{array}$ & $\begin{array}{l}\text { Neonatal } \\
\text { outcome }\end{array}$ \\
\hline 1 & $\mathrm{G}_{3} \mathrm{P}_{2} \mathrm{~L}_{2}$ & 39 & + & $\mathrm{N}$ & Oligo, IUD & $\mathrm{Y}$ & $\begin{array}{c}\text { Dengue } \\
\text { induced } \\
\text { hepatitis, } \\
\text { pulmonary } \\
\text { edema, and } \\
\text { death }\end{array}$ & IUD \\
\hline 2 & $\mathrm{G}_{4} \mathrm{P}_{3} \mathrm{~L}_{3}$ & $38+4$ & - & $\mathrm{Y}(\mathrm{PPH})$ & Oligo, LSCS & $\mathrm{Y}$ & $\mathrm{PPH}$, death & $\begin{array}{l}\text { NICU (neonatal } \\
\text { death- } \\
\text { meconium } \\
\text { aspiration } \\
\text { syndrome with } \\
\text { respiratory } \\
\text { failure) }\end{array}$ \\
\hline 3 & Primi & $34+4$ & + & $\mathrm{N}$ & PTVD & $\mathrm{Y}$ & $\begin{array}{c}\text { Preterm } \\
\text { delivery, } \\
\text { death }\end{array}$ & $\begin{array}{c}\text { NICU } \\
\text { (prematurity) }\end{array}$ \\
\hline 4 & Primi & 39 & + & $\mathrm{N}$ & Oligo, NVD & $\mathrm{N}$ & None & $\begin{array}{c}\text { NICU } \\
\text { (respiratory } \\
\text { distress) }\end{array}$ \\
\hline 5 & $\mathrm{G}_{2} \mathrm{P}_{1} \mathrm{~L}_{1}$ & $36+4$ & - & $\mathrm{N}$ & Oligo, NVD & $\mathrm{N}$ & None & Normal \\
\hline 6 & $\mathrm{G}_{3} \mathrm{P}_{2} \mathrm{~L}_{2}$ & $24+4$ & - & $\mathrm{N}$ & Oligo, NVD & $\mathrm{N}$ & None & Normal \\
\hline 7 & $\mathrm{G}_{3} \mathrm{P}_{2} \mathrm{~L}_{2}$ & 36 & - & $\mathrm{N}$ & $\begin{array}{l}\text { Oligo, FGR, } \\
\text { NVD }\end{array}$ & $\mathrm{N}$ & None & NICU (LBW) \\
\hline 8 & $\mathrm{G}_{2} \mathrm{P}_{1} \mathrm{~L}_{1}$ & 38 & + & $\mathrm{N}$ & Oligo, IUD & $\mathrm{N}$ & None & IUD \\
\hline 9 & $\mathrm{G}_{2} \mathrm{P}_{1} \mathrm{~L}_{1}$ & $17+4$ & - & $\mathrm{Y}(\mathrm{BPV})$ & $\begin{array}{c}\text { Spontaneous } \\
\text { abortion }\end{array}$ & $\mathrm{N}$ & Abortion & Abortion \\
\hline 10 & $\mathrm{P}_{2} \mathrm{~L}_{2}$ & Post LSCS & - & Y (hematoma) & $\begin{array}{c}\text { Rectus sheath } \\
\text { hematoma }\end{array}$ & $\mathrm{N}$ & $\begin{array}{c}\text { Hematoma } \\
\text { drainage }\end{array}$ & Normal \\
\hline 11 & $\mathrm{G}_{3} \mathrm{P}_{2} \mathrm{~L}_{1}$ & 40 & - & $\mathrm{Y}\left(2^{\circ} \mathrm{PPH}\right)$ & NVD & $\mathrm{N}$ & $2^{\circ} \mathrm{PPH}$ & $\begin{array}{c}\text { NICU } \\
\text { (meconium } \\
\text { aspiration) }\end{array}$ \\
\hline 12 & Primi & $34+2$ & - & Y (epistaxis) & NVD & $\mathrm{N}$ & Epistaxis & Normal \\
\hline 13 & Primi & 39 & - & $\mathrm{N}$ & $\begin{array}{l}\text { Forceps } \\
\text { delivery }\end{array}$ & $\mathrm{N}$ & $\mathrm{PPH}$ & Normal \\
\hline 14 & $\mathrm{G}_{2} \mathrm{P}_{1} \mathrm{~L}_{1}$ & $32+2$ & + & $\mathrm{Y}$ & PTVD & $\mathrm{N}$ & $\begin{array}{l}\text { Preterm } \\
\text { delivery }\end{array}$ & $\begin{array}{c}\text { NICU } \\
\text { (prematurity) }\end{array}$ \\
\hline 15 & $\mathrm{G}_{3} \mathrm{P}_{2} \mathrm{~L}_{2}$ & $37+5$ & - & $\begin{array}{l}\mathrm{N} \text { (bleeding } \mathrm{P} / \mathrm{R} \text {, } \\
\text { petechiae, and } \\
\text { subconjunctival } \\
\text { hemorrhage) }\end{array}$ & NVD & $\mathrm{N}$ & None & Normal \\
\hline 16 & Primi & $38+5$ & - & Y (hematuria) & IUD & $\mathrm{N}$ & None & IUD \\
\hline
\end{tabular}

G: gravidae, P: parity, L: living issue, VD: vaginal delivery, NVD: normal vaginal delivery, IUD: intrauterine death, PTVD: preterm vaginal delivery, PPH: postpartum hemorrhage, $2^{\circ} \mathrm{PPH}$ : secondary PPH, LBW: low birth weight, oligo: oligohydramnios, Y: yes, N: no, and NICU: neonatal intensive care unit.

pain, myalgia, arthralgia, rash, hemorrhagic manifestation, or leukopenia and positive serology or occurrence at a time of dengue outbreak was taken as the definition of dengue fever (DF). Dengue hemorrhagic fever (DHF) was classified as fever, hemorrhagic tendencies, thrombocytopenia, evidence of plasma leakage, association of hepatomegaly, and circulatory disturbances. Increase in serial hematocrit was taken as evidence of plasma leakage and ultrasound showing pleural effusion, ascites, and gall bladder edema were taken as supportive evidence. Dengue shock syndrome (DSS) was classified when DHF symptoms included rapid and weak pulse, narrow pulse pressure of less than $20 \mathrm{mmHg}$, and hypotension [2].

Patients were managed with antipyretics, adequate hydration, and blood product transfusion as necessary. Strict maternal and fetal surveillance were done to identify complications early. Platelet counts were done two to three times per day depending on clinical profile. Obstetric data, clinical, 
TABLE 2: Hematological indices and their variation during hospital stay.

\begin{tabular}{|c|c|c|c|c|c|c|c|}
\hline & \multicolumn{2}{|c|}{ Platelet count $\left(10^{3} / \mu \mathrm{L}\right)$} & \multicolumn{3}{|c|}{ Hematocrit (\%) } & \multirow{2}{*}{ Blood product transfusion } & \multirow{2}{*}{ Diagnosis } \\
\hline & Admission & Least & Admission & Maximum & Least & & \\
\hline 1 & 24 & 22 & 39 & 45 & 36 & $\mathrm{Y}$ & DSS \\
\hline 2 & 18 & 18 & 33 & 37.6 & 33 & $\mathrm{Y}$ & DSS \\
\hline 3 & 79 & 19 & 35 & 37 & 31.1 & $\mathrm{Y}$ & DSS \\
\hline 4 & 123 & 123 & 29 & 29 & 28 & $\mathrm{~N}$ & $\mathrm{DF}$ \\
\hline 5 & 40 & 24 & 23.4 & 30 & 23.4 & $\mathrm{Y}$ & DHF \\
\hline 6 & 45 & 45 & 30 & 31 & 28 & $\mathrm{~N}$ & $\mathrm{DF}$ \\
\hline 7 & 170 & 14 & 32.4 & 34.9 & 29 & $\mathrm{Y}$ & DHF \\
\hline 8 & 24 & 20 & 27.8 & 30 & 27 & $\mathrm{Y}$ & DHF \\
\hline 9 & 18 & 18 & 42 & 42 & 31 & $\mathrm{Y}$ & DHF \\
\hline 10 & 32 & 32 & 38 & 44 & 33 & $\mathrm{Y}$ & DHF \\
\hline 11 & 90 & 62 & 33 & 36 & 33 & $\mathrm{~N}$ & $\mathrm{DF}$ \\
\hline 12 & 190 & 43 & 32 & 39 & 32 & $\mathrm{~N}$ & DHF \\
\hline 13 & 76 & 76 & 29 & 33 & 29 & $\mathrm{~N}$ & $\mathrm{DF}$ \\
\hline 14 & 42 & 39 & 40 & 41 & 35 & $\mathrm{Y}$ & DHF \\
\hline 15 & 310 & 160 & 31 & 32 & 32 & $\mathrm{~N}$ & DF \\
\hline 16 & 16 & 16 & 39 & 39 & 30 & $\mathrm{Y}$ & DHF \\
\hline
\end{tabular}

laboratory parameters, and maternal and fetal outcomes of women are depicted in Table 1 . The platelet count and hematocrit variation of the cases are shown in Table 2.

\section{Results and Discussion}

A majority of women were unbooked and presented with classical symptoms of high grade fever, myalgia, headache, and rashes to the emergency room. All women were young, age ranging from 22 to 32 years; mean age was 25 years. Out of 16 cases 13 presented in third trimester and two in second trimester and one was referred after LSCS was done in another hospital. She presented with large rectus sheath hematoma following caesarean section needing surgical exploration and drainage. None of the women had any underlying diseases or comorbidity except one (\#case 5) who had moderate anemia on admission.

In the current study, eight women (50\%) had dengue haemorrhagic fever (DHF). Ten women required transfusion to maintain platelet count in desired range; on average each woman required six platelet transfusions. Most women had low platelet counts at the time of admission (mean $\left.81,000 / \mathrm{mm}^{3}\right) ; 62 \%$ had platelets less than $50,000 / \mathrm{mm}^{3}$. Platelet count less than $30,000 / \mathrm{mm}^{3}$ was seen in dengue shock syndrome with increased risk of bleeding manifestations. A Sri Lankan case series reported 10 women with DHF but only eight required platelet transfusion [5]. Three women succumbed to dengue shock syndrome in current study with a mortality rate of $18 \%$ compared to $3-5 \%$ case fatality rate in rest of the India in nonpregnant population [2]. In another study done in India by Agrawal et al. maternal mortality rate was $12 \%[3]$.

Symptomatic dengue infection may increase the risk of preterm labour and, hence, low birth weight (LBW) as suggested by previous studies $[3-5,11]$. In the study under discussion two women had preterm delivery (12\%). Most women presented near term $(n=10)$ and of the four who presented in early gestation two had preterm labour. Preterm births reported were as low as $11 \%$ in the French Guiana study versus $41 \%$ in the study by Basurko et al. $[4,11]$. One woman had spontaneous abortion (6\%) and seven (43\%) pregnancies were complicated with oligohydramnios in present study compared to $52 \%$ rate of oligohydramnios from a study in India by Agrawal et al. [3].

Bleeding manifestations were seen in seven women and three (19\%) had postpartum hemorrhage (PPH) ( $\# 2,11$, and 13). One of them was diagnosed as DSS and the other two were diagnosed as DF. Both women diagnosed as DF had atonic PPH. One of them had PPH 48 hours following the delivery. Both the patients did not have any other bleeding manifestations and signs of plasma leakage hence were classified as only DF. In retrospective study by Basurko et al. on 53 pregnant women, $\mathrm{PPH}$ was reported in $10 \%$ of the cases compared to $19 \%$ in our study [4].

There were six neonatal intensive care unit (NICU) admissions (37\%), three intrauterine deaths (IUD) (18\%), and one neonatal death $(6 \%)$. One neonatal death was caused by meconium aspiration syndrome. None of the neonates in our study showed symptoms of dengue such as thrombocytopenia, anemia, fever, or bleeding manifestations. Diagnostic tests for dengue were not routinely done for asymptomatic neonates. There are a number of studies on poor fetal outcome following dengue infection in parturient females. Vertical transmission of dengue has also been reported $[4,5]$. Basurko et al. reported 5.3\% vertical transmission rate in their study [4]. They also reported IUD rate of $3.8 \%$ and neonatal death rate of $1.9 \%$ while Carles et al. also reported high fetal death rate $[4,12]$.

Pregnant women with dengue fever should be considered for admission early because of its unpredictable course. 
Maintaining normothermia and adequate hydration should be the goal. Low platelet counts should be taken as a marker of severity of the disease. Oligohydramnios is an ominous sign when seen concomitantly with dengue infection because the high fetal mortality was associated with it. The exact cause of oligohydramnios is not known but dehydration associated with dengue fever may be contributory. Hydration should be maintained by encouraging oral intake of oral rehydration solution (ORS), fruit juice, and other fluids containing electrolytes and glucose for replacing losses from fever and associated vomiting [2]. Dengue fever does not warrant any active obstetrical intervention.

Another dilemma faced was in differentiating dengue infection from HELLP syndrome as both have somewhat similar laboratory parameters; however serology will help clinch the diagnosis. Careful examination and high index of suspicion by the treating obstetrician are required to diagnose and manage such cases especially during epidemics.

\section{Conclusions}

Our knowledge on effect of dengue on pregnancy is somewhat limited. In this study dengue was associated with high maternal and fetal morbidity and mortality. Dengue needs early diagnosis and prompt treatment to avoid its adverse outcomes. Pregnant women should avoid travel to dengue afflicted regions. Vector control methods should be employed during seasonal outbreaks. Awareness programs and medical education programs on the management of the dengue in pregnancy should be initiated especially during outbreaks to provide quality care.

\section{Competing Interests}

The authors declare that there are no competing interests regarding the publication of this paper.

\section{References}

[1] J. G. Rigau-Pérez, G. G. Clark, D. J. Gubler, P. Reiter, E. J. Sanders, and A. V. Vorndam, "Dengue and dengue haemorrhagic fever," The Lancet, vol. 352, no. 9132, pp. 971-977, 1998.

[2] WHO Dengue: Guidelines for Diagnostics, Treatment, Prevention and Control, World Health Organization, Geneva, Switzerland, 2009.

[3] P. Agrawal, R. Garg, S. Srivastava, U. Verma, and R. Rani, "Obstetrics and gynecology pregnancy outcome in women with Dengue infection in northern India," Indian Journal of Clinical Practice, vol. 24, no. 11, p. 1053, 2014.

[4] C. Basurko, G. Carles, M. Youssef, and W. E. L. Guindi, "Maternal and foetal consequences of dengue fever during pregnancy," European Journal of Obstetrics Gynecology \& Reproductive Biology, vol. 147, no. 1, pp. 29-32, 2009.

[5] S. Kariyawasam and H. Senanayake, "Dengue infections during pregnancy: case series from a tertiary care hospital in Sri Lanka," Journal of Infection in Developing Countries, vol. 4, no. 11, pp. 767-775, 2010.

[6] I. Dale Carroll, S. Toovey, and A. V. Gompel, "Dengue fever and pregnancy-a review and comment," Travel Medicine and Infectious Disease, vol. 5, no. 3, pp. 183-188, 2007.
[7] U. Chotigeat, S. Kalayanarooj, and A. Nisalak, "Vertical transmission of dengue infection in thai infants: two case reports," Journal of the Medical Association of Thailand, vol. 86, supplement 3, pp. S628-S632, 2003.

[8] J. K. Chye, C. T. Lim, K. B. Ng, J. M. H. Lim, R. George, and S. K. Lam, "Vertical transmission of dengue," Clinical Infectious Diseases, vol. 25, no. 6, pp. 1374-1377, 1997.

[9] S. L. C. Maroun, R. C. C. Marliere, R. C. Barcellus, C. N. Barbosa, J. R. M. Ramos, and M. E. L. Moreira, "Case report: vertical dengue infection," Jornal de Pediatria, vol. 84, no. 6, pp. 556559, 2008.

[10] L. E. Fatimil, A. H. Mollah, S. Ahmed, and M. Rahman, "Vertical transmission of dengue: first case report from Bangladesh," The Southeast Asian Journal of Tropical Medicine and Public Health, vol. 34, no. 4, pp. 800-803, 2003.

[11] E. E. Friedman, F. Dallah, E. W. Harville et al., "Symptomatic dengue infection during pregnancy and infant outcomes: a retrospective cohort study," PLoS Neglected Tropical Diseases, vol. 8, no. 10, article e3226, 2014.

[12] G. Carles, A. Talarmin, C. Peneau, and M. Bertsch, "Dengue fever and pregnancy. A study of 38 cases in French Guiana," Journal de Gynécologie, Obstétrique et Biologie de la Reproduction (Paris), vol. 29, pp. 758-762, 2000. 


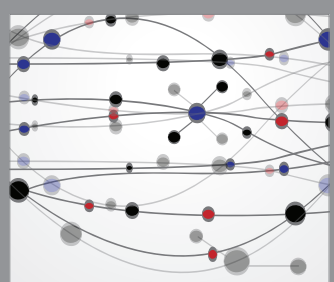

The Scientific World Journal
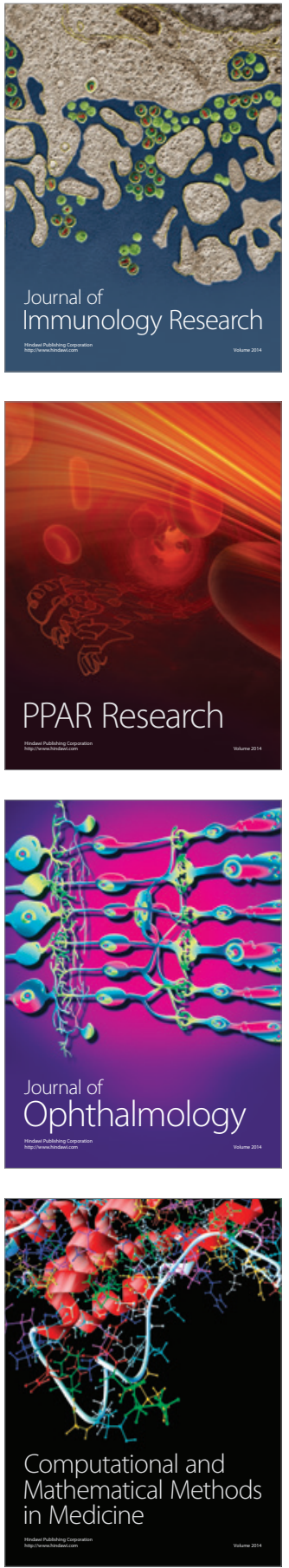

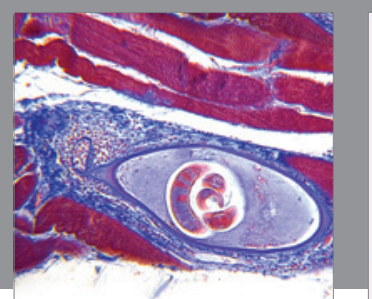

Gastroenterology Research and Practice

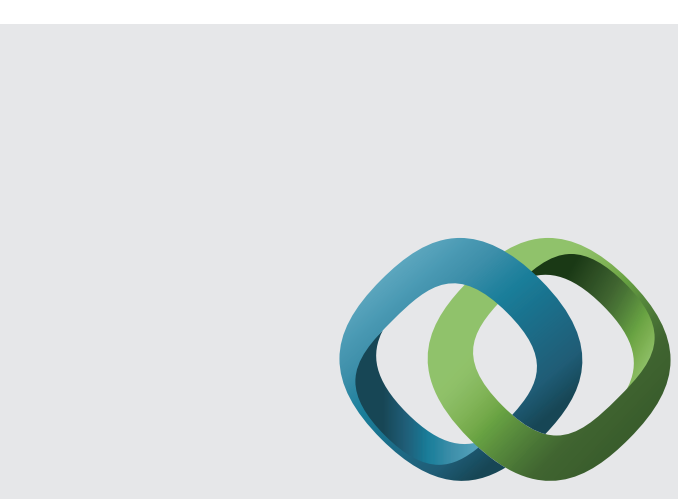

\section{Hindawi}

Submit your manuscripts at

http://www.hindawi.com
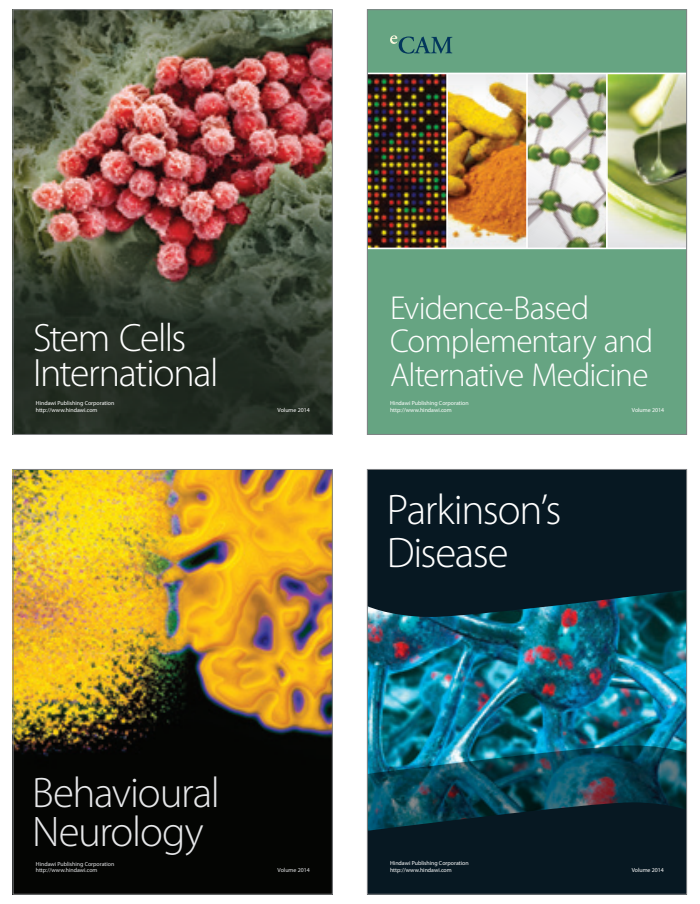
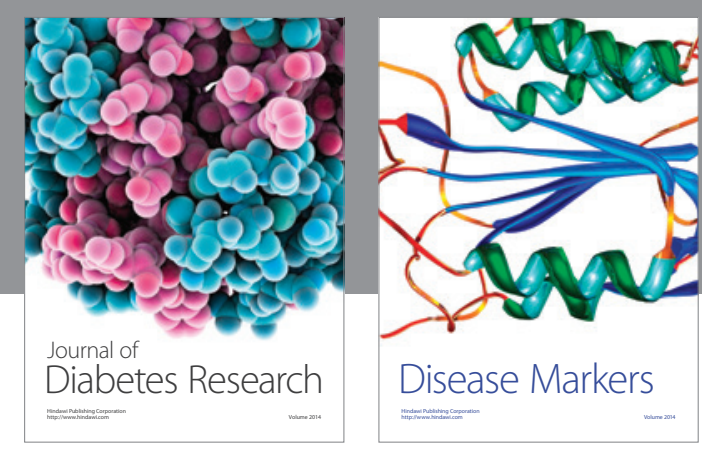

Disease Markers
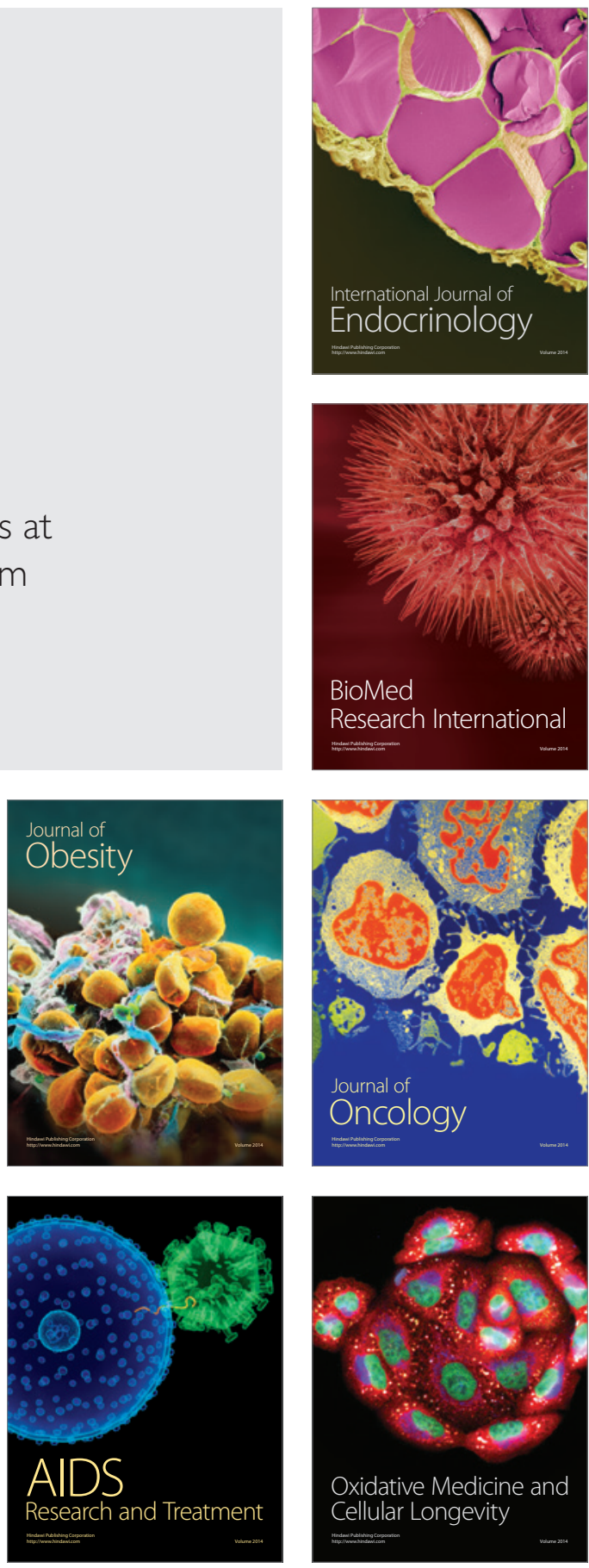